\title{
The association between pulmonary artery systolic pressure and mechanical ventilator weaning success in critically ill patients with COVID-19.
}

\author{
Luo Yong-Fang ${ }^{1}$, Zeng Jia-Hui ${ }^{1}$, Huang Kun ${ }^{1}$, Luo Ting-Ting ${ }^{1}$, Zhang $\mathrm{Yu}^{1}$, Xu Wei-Tao ${ }^{1}$, \\ Zhou Dan ${ }^{1}$, Wu Wei-Bo ${ }^{1}$, Zhang Jin², Duan Jing-Jing ${ }^{2}$, Feng Yong-Wen ${ }^{3}$, Fu Ying-Yun ${ }^{4}$, \\ Wen Jun-Min ${ }^{5}$, Gao Hong ${ }^{1}$, and Cheng Feng ${ }^{1}$ \\ ${ }^{1}$ The Third People's Hospital of Shenzhen \\ ${ }^{2}$ Nanchang University School of Science \\ ${ }^{3}$ Shenzhen Second People's Hospital \\ ${ }^{4}$ Shenzhen People's Hospital \\ ${ }^{5}$ Fuwai HospitalChinese Academy of Medical Sciences
}

October 5, 2020

\begin{abstract}
To explore the association between pulmonary artery systolic pressure (PASP) and success to wean from mechanical ventilation in COVID-19 patients. Thirty-four critically ill COVID-19 patients who had been placed on ventilator support were enrolled in this retrospective study and were divided into two groups: the weaning success group (group I) and the weaning failure group (group II). Physical examination, echocardiography, and routine laboratory test data were collected. The levels of troponin I, myoglobin, interleukin 6, and D-dimer were significantly lower in group I than in group II. The interventricular septum end-diastolic thickness (IVSd) was thicker (14.00 [13.50-15.00] mm) and the PASP was higher (61.63 $\pm 13.53 \mathrm{mmHg})$ in group II. Logistic regression analysis showed that D-dimer and IVSd were factors associated with ventilator weaning. The odds ratio (OR) of D-dimer was 0.81 (95\% CI 0.69-0.95) and the OR of IVSd was 0.16 (95\% CI 0.045-0.59). Cox analysis revealed that the adjusted hazard ratio (HR) for a 1-mmHg increase in PASP was 0.94 (95\% CI 0.90-0.97) for weaning events $(\mathrm{P}<0.001)$. PASP was negatively correlated with the $\mathrm{PaO} 2: \mathrm{FiO} 2(\mathrm{r}=-0.48, \mathrm{P}<0.01)$. A receiver operating characteristic curve showed that the best cutoff value of PASP for ventilator weaning was $39.50 \mathrm{mmHg}$, with a sensitivity of $100 \%$ and a specificity of $96.15 \%$ PASP was an independent risk factor for the difficulty of weaning severely ill COVID-19 patients off mechanical ventilation. PASP can be used as one of the indicators for evaluating the condition of COVID-19 patients.
\end{abstract}

\section{Introduction}

Coronavirus disease-2019 (COVID-19) has resulted in considerable morbidity and mortality worldwide since December 2019. Clinical features of COVID-19 patients have recently been well described, and the existence of heart injury in these patients has been confirmed. A previous study reported that $12 \%$ of COVID-19 patients had related acute heart injury, accompanied by decreased ejection fraction (EF) and elevated troponin I (TnI)1. In another case series, the percentage of patients with acute cardiac injury and arrhythmia was even higher in severe COVID-19 patients (22.2\% and $44.4 \%$, respectively)2. It has been reported that cardiac injuries are common in critically ill patients with COVID-193; some case series reported a peak value of TnI exceeding 40-fold the normal value4. Nevertheless, information on cardiac injury in patients affected by COVID-19 is relatively limited at present.

There is a need to investigate prognostic markers for severe COVID-19 patients to provide important in- 
formation for early therapeutic strategies. Echocardiography is a convenient and repeatable approach that allows control of cross-infection in the hospital and is suitable to examination in the ICU; thus, echocardiography has become an indispensable examination method for evaluating COVID-19 patients in ICU. This study therefore attempted to use echocardiographic measurements to predict the chance of weaning from mechanical ventilation in patients with critical COVID-19. Herein, we retrospectively analyzed clinical data from Shenzhen Third People's Hospital, China, to explore the potential association between pulmonary artery systolic pressure (PASP), determined echocardiographically, and success to wean from mechanical ventilation in critically ill patients with COVID-19.

\section{Methods}

\section{Participants}

This cohort study was conducted from January 11 to April 10, 2020, at Shenzhen Third People's Hospital in Guangdong, China. The Shenzhen Third People's Hospital was designated by the Shenzhen municipal government to be responsible for the treatment of COVID-19 patients in the city since January 2020. All patients included in this study had laboratory-confirmed COVID-19 and had received mechanical ventilation support. Participants were divided into two groups depending on whether they had been successfully weaned off the ventilator. Weaning failure was defined by failure of spontaneous breathing trials or a need for reintubation or non-invasive ventilation, or death within $48 \mathrm{~h}$ after extubation5. Patients in severe type should be consistent with any of the following items: (1) respiratory rate higher than 30 times / min; (2) fingertip blood oxygen saturation less than $93 \%$ in resting state; (3) partial arterial oxygen pressure/ fraction of inspiration oxygen(PaO2:FiO2) less than $300 \mathrm{mmHg}$. Patients with one of the three conditions are considered as critical type: Any of the following items: (1) respiratory failure, and requires mechanical ventilation; (2) shock; (3) multiple organ failure, which requires intensive care unit monitoring and treatment. The study was approved by the Ethics Committee of Shenzhen Third People's Hospital (No.2020-157). Informed consent was obtained before retrospective data collection. A copy of the written consent is available for review by the Editor of this journal.

Data collection

Patient demographics, medical history, clinical records, laboratory tests, and ventilator data were collected and analyzed. Blood gas analysis and echocardiography were performed at the same day. The associations between cardiac parameters and ventilator treatment were analyzed. The patients were followed up from the day that mechanical ventilation was implemented to assist breathing to the day of weaning from ventilation (1-30 days). Death was classified as a failure to wean the patient from the ventilator.

\section{Echocardiographic assessment}

All examinations are performed by senior doctors according to the standard procedure within the first 24 hours in ICU. Mindray-M9 portable color Doppler ultrasound and a P4-2s probe $(2-4 \mathrm{MHz})$ were used to perform echocardiography. Measurements of interventricular septal end-diastolic thickness (IVSd), left ventricular end-diastolic dimension (LVDd), left ventricular ejection fraction (LVEF), pulmonary artery systolic pressure (PASP), and left ventricular diastolic function were obtained from each echocardiographic examination. With the guidance of tricuspid regurgitation detected by color Doppler, continuous wave Doppler was used to measure the maximum velocity of tricuspid regurgitation and the tricuspid valve regurgitation pressure gradient (TRPG). The PASP was estimated by TRPG plus right atrial pressure (RAP). The right ventricular inflow tunnel section, parasternal short axis, parasternal four chamber, apical four-chamber or five-chamber sections were selected according to the clarity of the image in each patient. According to the collapsibility of inferior vena cava (IVC), RAP was valued as 5,10 , or $15 \mathrm{mmHg}$, respectively6. In the case of mechanical ventilator usage, central venous pressure (CVP) was considered to be equal to RAP. The highest PASP value was used for statistical analysis. In order to ensure accuracy and repeatability, the echocardiographic data were collected by two independent senior doctors.

\section{Statistical analysis}


Statistical analysis was performed using SPSS version 26.0 (IBM SPSS Inc., Chicago, IL). Continuous variables are presented as mean \pm standard deviation or median [interquartile range] for normal and non-normally distributed data, respectively. Categorical variables are presented as absolute values and percentages. Means were compared using the independent group $t$-test or the Mann-Whitney $\mathrm{U}$ test (depending on data normality) for continuous variables and the $\chi^{2}$ test (two-sided Fisher's exact test) for categorical variables. Logistic regression analysis was used to screen the factors related to the outcome and meaningful indicators for the next step of Cox multivariate regression analysis. Cox proportional hazards models were used to analyze the independent effect of PASP on ventilator treatment. Spearman's correlation analysis was used to investigate the correlation of PASP with oxygen pressure/ fraction of inspiration oxygen ( $\mathrm{PaO} 2: \mathrm{FiO} 2)$. The cumulative survival curve of ventilator weaning was estimated using the Kaplan-Meier product-limit estimation method. A receiver operating characteristic (ROC) curve was drawn to evaluate the predictive value of PASP for weaning outcome. $P<0.05$ was considered to indicate statistical significance.

\section{Results}

Presenting characteristics, laboratory tests, and echocardiographic parameters

In this study, 34 patients with COVID-19 in Shenzhen Third People's Hospital were retrospectively enrolled; these included 19 critical ill patients and 15 seriously ill patients. All the patients were treated with ventilation therapy; 16 patients received non-invasive ventilator support, with an average duration of ventilation of $7.3 \pm 4.5$ days; 16 patients received both non-invasive and invasive ventilation support (average duration, non-invasive: $3.8 \pm 2.9$ days vs invasive: $15.3 \pm 7.3$ days); 2 patients received invasive ventilation support from the beginning of follow-up (25.0 \pm 7.1 days). There were $3(8.8 \%)$ deaths during follow-up.

According to whether patients were successfully weaned from the ventilator, they were divided into two groups: the weaning success (group I) and weaning failure group (group II). There were 26 patients in group I, including 15 males and 11 females and 8 patients in group II, all of whom were male. Patients in group II were significantly older than those in group I $(P<0.047)$.

The myocardial injury markers were significantly higher in group II than group I (Table 1). The interleukin 6 (IL-6) and D-dimer levels were also significantly higher in group II than in group I $(P=0.01$ and $P=0.002$ , respectively). The IVSd was thicker $(P<0.001)$ and, importantly, the PASP was higher $(P<0.01)$ in group II than in group I $(61.63 \pm 13.53 \mathrm{mmHg}$ vs $24.27 \pm 7.70 \mathrm{mmHg})$ (Table 1). Other characteristics, such as body temperature, comorbidities history, creatine kinase, procalcitonin (PCT), C-reactive protein (CRP), brain natriuretic peptide, OI, LVEF, and LVDd showed no insignificance between the two groups (Table 1). The line chart of PASP in the two groups showed that the average PASP in group II was significantly higher than that in group I from the $28^{\text {th }}$ day of follow-up (Figure 1).

Predictors of ventilator weaning

The parameters that were statistically significant in univariate analysis, including age, IL-6, D-dimer, TnI, IVSd, and PASP were further evaluated in logistic regression analysis to explore whether they were related to ventilator weaning. The results showed that D-dimer and IVSd may be statistically significant risk factors for the failure of ventilator weaning. The OR of D-dimer was 0.81 (95\% CI 0.69-0.95) and the OR of IVSd was 0.16 (95\% CI 0.045-0.59) (Table 2).

Cox hazard regression analysis

In Cox multivariate regression model analysis, with ventilator weaning as outcome, time was defined as the number of days of mechanical respiratory assistance, the start time was the time of admission to the ICU, and the follow-up time was 30 days. Regression modeling results are shown in Table 3. In logistic regression analysis, PASP had not shown an association with the outcome. However, based on our clinical experience, PASP was closely related to clinical performance and pulmonary imaging changes in patients; thus, we entered PASP into the Cox analysis (model 1). As described above, D-dimer and IVSd could be used as predictors of ventilator weaning when added to the model respectively for correction (models $2 \& 3$ ), but these models revealed no statistical significance. Our Cox hazard regression analysis results demonstrated 
that PASP was a critically important indicator, but had an independent effect on events after adjusting for potential confounders, including time. The adjusted hazard ratio (HR) for ventilator weaning for a 1-mmHg increase in PASP was 0.94 (95\% CI 0.90-0.97) ( $\left.\mathrm{P}_{j} 0.001\right)$.

Correlation of PASP with

\section{PaO2:FiO2}

In present study, PASP was negatively correlated with $\mathrm{PaO} 2$ :FiO2 (Spearman's $\mathrm{r}=-0.48, \mathrm{P}<0.01$ ) (Figure 2).

Cumulative survival curve for weaning from the ventilator

The cumulative survival rate curve for ventilator weaning is shown in Figure 3.

Receiver operator characteristic curve for prediction in weaning from ventilator

The ROC curve was analyzed by selecting the maximum pulmonary pressure during follow-up. The area under the curve (AUC) for ventilator weaning was 0.99. The best cutoff value for PASP for predicting the success of ventilator weaning was $39.50 \mathrm{mmHg}$, yielding a sensitivity of $100 \%$ and a specificity of $96.15 \%$ (Figure 4).

\section{Discussion}

This study evaluated the prognostic value of pulmonary artery systolic pressure (PASP) in predicting outcomes of weaning patients with COVID-19 from mechanical ventilation. The adjusted HR for a 1-mmHg increase in PASP was 0.94 (95\% CI 0.90-0.97) for weaning $(\mathrm{P}<0.001)$, and PASP was negatively correlated with the $\mathrm{PaO} 2$ : $\mathrm{FiO} 2(\mathrm{r}=-0.48, \mathrm{P}<0.01)$. The appropriate cutoff value of PASP for ventilator weaning was $39.50 \mathrm{mmHg}$, with a sensitivity of $100 \%$ and a specificity of $96.15 \%$

Epidemiological studies of COVID-19 have shown that the mortality rate of critical patients is $49 \%$, accounting for $5 \%$ of confirmed patients7. Recent studies have revealed that the severity of the disease is an independent predictor of a poor prognosis8. The effective and appropriate treatment for critically ill patients seems to be a key for decreasing mortality. Successful ventilator weaning often indicates a good prognosis. At present, the conditions for clinical discontinuation from mechanical ventilation are the minute ventilation volume, maximum inspiratory pressure, respiratory frequency, rapid shallow breathing index, 0.1-second airway closure pressure and so on9, 10. However, a previous study showed that attempts at extubation failed in approximately $20 \%$ of mechanically ventilated patients, and these patients spent more than $40 \%$ of the total mechanical ventilation time in the overall weaning process11. Improper extubation time leads to hypoxemia, and can even lead to a need for tracheal reintubation. On the other hand, prolonged mechanical ventilation time increases the risk of related complications (such as infection, gastrointestinal bleeding, deep vein thrombosis, etc.)12. Due to the recurrent nature of the disease symptoms, many patients had met the clinical indicators and were temporarily weaned off the ventilator, but then required mechanical ventilation again. Therefore, in order to ascertain the correct timing for weaning patients off the ventilator, more accurate and specific predictors appropriate to the characteristics of COVID-19 are needed.

In this study, we found statistically significant differences in biochemical indicators (IL-6 and D-dimer) between the weaning success and weaning failure groups. The former indicated that the immune response of patients who failed to be weaned off the ventilator was stronger, and the latter reflected the fibrinolytic dysfunction in these patients. The levels of PCT and CRP were higher than the normal values, without significant differences between the two groups, indicating that both groups showed enhanced inflammation. In terms of myocardial injury markers, the levels of TnI and myoglobin were significantly higher in group II than in group I, indicating that patients in whom ventilator weaning failed had more serious heart injury.

We also found that patients who failed to wean off the ventilator had a thicker interventricular septum and a higher PASP on echocardiography, which was consistent with a previous study13. The thickened interventricular septum may be related to the cytokine storm associated with COVID-19. In our study, the 
levels of IL-6 were significantly elevated in both groups, and was higher in patients who were not successfully weaned from the ventilator. The cytokine storm may increase the permeability of the vascular wall and could lead to myocardial edema14, 15. In addition, hypoxia can also lead to an early and substantial inflammatory response and cell damage16, and may also lead to some myocardial thickening and edema.

Among the echocardiographic measurements, PASP appeared to be most related to the severity of the patients' condition. Our research showed that PASP had a moderate correlation with the OI, which indicated that PASP could partly reflect the lung condition of COVID-19 patients. The result of Cox analysis showed that PASP was an independent risk factor of ventilator evacuation in critically ill COVID-19 patients. For every $1 \mathrm{mmHg}$ increase in PASP, the risk of weaning from the ventilator was 0.94 times higher, even after adjusting for other risk factors. The ROC curve showed that the best cutoff value for PASP for predicting the success of weaning off the ventilator was $39.50 \mathrm{mmHg}$, with a sensitivity of $100 \%$ and a specificity of 96.15\% ( $\mathrm{P}<0.0001)$, and an AUC is 0.99, which showed the good power of the test. We suggest that PASP and other criteria for ventilator weaning should be considered together. In critically ill patients, if the PASP is higher than $39.5 \mathrm{mmHg}$, ventilator weaning should not be considered.

Other studies have also confirmed that PASP is related to prognosis in patients with cardiovascular disease. One study showed that when PASP $>45 \mathrm{mmHg}$, the prognosis of acute decompensated heart failure (ADHF) was poor; early right ventricular dilatation or dysfunction suggested a poor prognosis, while LVEF or left ventricular diastolic function could not predict prognosis17.

There are many reasons for the increased pulmonary artery pressure in critically ill COVID-19 patients. Anatomic reasons include inflammation of pulmonary arterioles involved in bronchitis and the surrounding inflammation, accompanied by thickening, spasm, or fibrosis of the blood vessel walls. Functional reasons include pulmonary vasoconstriction and spasm caused by a neurohumoral mechanism, such as hypoxia and respiratory acidosis, which increase pulmonary circulatory resistance. In addition, secondary injury (emphysema) is caused by long-term mechanical ventilation, which indirectly causes the rise of PASP. Computed tomography angiography of one case in our study confirmed that the spasticity and thrombus of pulmonary arterioles caused the PASP elevation and that PASP fluctuates during anticoagulation and vasodilation treatment. In addition to the pulmonary vasospasm caused by the pathological changes in the lung itself, and the decrease in pulmonary vascular compliance caused by pulmonary fibrosis, the accompanying cardiac diseases also have a significant impact. Acute virus replication in the myocardium and secondary inflammatory responses can lead to acute myocardial injury, some of which can lead to left ventricular systolic dysfunction and myocardial swelling18, that is, ADHF, which can aggravate the pulmonary congestion. With the progression in the disease course, the pulmonary artery pressure of these patients increased gradually, which increased the burden of the right heart. In the present study, two of the three patients who died showed a sudden decrease in right heart function and pulmonary artery pressure several days before death. Therefore, PASP elevation is a comprehensive indicator of the interaction between systemic inflammation and pathogen-mediated cardiovascular and respiratory system damage, which may be the mechanism underlying the worse outcomes.

PASP measurements in this study could have been influenced by various factors, such as nitric oxide (NO) treatment and increased blood volume due to fluid retention. NO gas is soluble in water and easily passes through a lipid membrane. NO can relax vascular smooth muscle, inhibit platelet aggregation, affect leukocyte chemotaxis, control inflammation, ease pain, regulate immunity, eliminate oxygen free radicals, and protect the gastric mucosa. NO can combine with hemoglobin in red blood cells and acts as a potent vasodilator19. Three of our patients were treated with NO, which temporarily reduced but did not reverse PASP.

Furthermore, hypoxia and hypercapnia can cause sympathetic nerve excitability, renal vasoconstriction, and decreased renal blood flow, resulting in water and sodium retention. The increased blood volume can lead to increased pulmonary circulation resistance. However, the patients in this study underwent strict volume management to avoid fluid retention as far as possible, and the left atrial diameters were rarely enlarged, as measured by echocardiography. A previous study also showed no significant association between 
echocardiographic indices of filling pressures and weaning failures20. Therefore, PASP in this study was less affected by pre-pulmonary circulation load.

PASP did not significantly associated with success of ventilator weaning in the logistic regression analysis; however, it showed an association with the outcome in Cox regression analysis. This may be explained by the addition of the time variable, for correction, in the Cox regression analysis; this result shows that continuous pulmonary hypertension, rather than temporary changes, may be more strongly related to the prognosis.

In this study, estimation of pulmonary artery pressure was based on TRPG plus RAP. The literature on mechanical ventilation indicates that CVP is equivalent to RAP; hence, we used CVP instead of RAP. For spontaneous respiration, we used the American Society of Echocardiography guidelines for estimation; sonographic measurement of the IVC diameter and collapsibility was used to estimate RAP21. In terms of the effect of mechanical ventilation on right atrial pressure, high positive end-expiratory pressure (PEEP; 8-10 $\mathrm{cmH} 2 \mathrm{O}$ ) will affect the flow from the right heart to the left heart. However, the hemodynamics of the heart mainly depend on changes in the internal pressure of the heart, and our patients' PEEPs were generally below 8-10 cmH2O during mechanical ventilation; thus, the increase in the external pressure likely had little effect22.

\section{Limitations}

There are some limitations in the present study. First, some data, such as echocardiography data, electrocardiography data, and cytokine level measurements, were lacking from clinical examinations of patients in isolation wards or the ICU, which limits the determination of potential mechanisms of cardiac injury. Second, there may be some errors in measurement of TRPG due to different operators or poor image quality. Mediastinal emphysema occurred in four patients and PASP could not be measured. Third, due to the limited number of COVID-19 patients in a single center, the sample size of this study was relatively small. Therefore, data from larger populations and multiple centers are required to confirm the correlation of PASP with outcomes in COVID-19 patients.

\section{Conclusions}

Cardiac injury is a common condition among patients with COVID-19. PASP may comprehensively reflect lung injury. Echocardiography could be used as a means for evaluation of the severity of the disease and may be associated with whether the patient is likely to be weaned off the ventilator successfully. Thus, PASP levels in critically ill COVID-19 patients may help to identify patients with poor prognoses.

\section{Acknowledgements}

We thank all the staffs working at the front-line to battle against SARS-CoV-2. We show our respect to those who have sacrificed in this war.

\section{Author Contribution}

YFL and CF had full access to all of the data and take responsibility for the integrity and accuracy of the data.

Concept and design: LL, CF.

Acquisition of data: YFL, JHZ, KH, TTL, YZ, WTX, DZ.

Analysis or interpretation of data: YFL and JHZ.

Drafting of the manuscript: JHZ and YFL.

Critical revision of the manuscript for important intellectual content: WBW, YWF, YYF, JMW, YXL.

Polish of manuscript: JZ, JJD.

Supervision: HG, LL, and CF. 
All authors read and approved the final manuscript.

\section{Source of Funding}

This manuscript is funded by Guangdong Medical Research Fund (The Grant B2019163).

\section{Disclosures}

\section{None.References}

1. Huang C, Wang Y, Li X, et al. Clinical features of patients infected with 2019 novel coronavirus in Wuhan, China. Lancet. Jan 242020.

2. Wang $\mathrm{D}, \mathrm{Hu} \mathrm{B}, \mathrm{Hu} \mathrm{C}$, et al. Clinical Characteristics of 138 Hospitalized Patients With 2019 Novel Coronavirus-Infected Pneumonia in Wuhan, China. JAMA. Feb 72020.

3. Shi S, Qin M, Shen B, et al. Association of Cardiac Injury With Mortality in Hospitalized Patients With COVID-19 in Wuhan, China.JAMA Cardiol. Mar 252020.

4. Hui H, Zhang Y, Yang X, et al. Clinical and radiographic features of cardiac injury in patients with 2019 novel coronavirus pneumonia. medRxiv. 2020:2020.2002.2024.20027052.

5. Boles JM, Bion J, Connors A, et al. Weaning from mechanical ventilation. Eur Respir J. May 2007;29(5):1033-1056.

6. Beigel R, Cercek B, Luo H, Siegel RJ. Noninvasive evaluation of right atrial pressure. J Am Soc Echocardiogr. Sep 2013;26(9):1033-1042.

7. Rothan HA, Byrareddy SN. The epidemiology and pathogenesis of coronavirus disease (COVID-19) outbreak. J Autoimmun. Feb 26 2020;109:102433.

8. Fang Z, Yi F, Wu K, et al. Clinical Characteristics of Coronavirus Pneumonia 2019 (COVID-19): An Updated Systematic Review.medRxiv. 2020:2020.2003.2007.20032573.

9. Ferrari G, De Filippi G, Elia F, Panero F, Volpicelli G, Apra F. Diaphragm ultrasound as a new index of discontinuation from mechanical ventilation. Crit Ultrasound J. 2014;6(1):8.

10. Yang KL, Tobin MJ. A prospective study of indexes predicting the outcome of trials of weaning from mechanical ventilation. $N$ Engl J Med. May 23 1991;324(21):1445-1450.

11. Zambon M, Greco M, Bocchino S, Cabrini L, Beccaria PF, Zangrillo A. Assessment of diaphragmatic dysfunction in the critically ill patient with ultrasound: a systematic review. Intensive Care Med. Jan 2017;43(1):29-38.

12. DiNino E, Gartman EJ, Sethi JM, McCool FD. Diaphragm ultrasound as a predictor of successful extubation from mechanical ventilation. Thorax. May 2014;69(5):423-427.

13. Deng Q, Hu B, Zhang Y, et al. Suspected myocardial injury in patients with COVID-19: Evidence from front-line clinical observation in Wuhan, China. Int J Cardiol. Apr 82020.

14. Channappanavar R, Perlman S. Pathogenic human coronavirus infections: causes and consequences of cytokine storm and immunopathology. Semin Immunopathol. Jul 2017;39(5):529-539.

15. Huang KJ, Su IJ, Theron M, et al. An interferon-gamma-related cytokine storm in SARS patients. $J$ Med Virol. Feb 2005;75(2):185-194.

16. Wu J, Stefaniak J, Hafner C, et al. Intermittent Hypoxia Causes Inflammation and Injury to Human Adult Cardiac Myocytes. Anesth Analg. Feb 2016;122(2):373-380.

17. Palazzuoli A, Ruocco G, Evangelista I, De Vivo O, Nuti R, Ghio S. Prognostic Significance of an Early Echocardiographic Evaluation of Right Ventricular Dimension and Function in Acute Heart Failure.J Card Fail. Jan 112020. 
18. Edwards S, Small JD, Geratz JD, Alexander LK, Baric RS. An experimental model for myocarditis and congestive heart failure after rabbit coronavirus infection. J Infect Dis. Jan 1992;165(1):134-140.

19. Kumar S, Singh RK, Bhardwaj TR. Therapeutic role of nitric oxide as emerging molecule. Biomed Pharmacother. Jan 2017;85:182-201.

20. Bouhemad B, Mojoli F, Nowobilski N, et al. Use of combined cardiac and lung ultrasound to predict weaning failure in elderly, high-risk cardiac patients: a pilot study. Intensive Care Med.Mar 2020;46(3):475484.

21. Ciozda W, Kedan I, Kehl DW, Zimmer R, Khandwalla R, Kimchi A. The efficacy of sonographic measurement of inferior vena cava diameter as an estimate of central venous pressure. Cardiovasc Ultrasound. Aug 20 2016;14(1):33.

22. Cabello B, Thille AW, Roche-Campo F, Brochard L, Gomez FJ, Mancebo J. Physiological comparison of three spontaneous breathing trials in difficult-to-wean patients. Intensive Care Med. Jul 2010;36(7):11711179 .

Table 1. Presenting characteristics, Laboratory and echocardiographic parameters

\begin{tabular}{|c|c|c|c|c|}
\hline & Total $(\mathrm{n}=34)$ & Group I $(n=26)$ & Group II $(n=8)$ & $P$ value \\
\hline$\overline{\text { Age, median [IQR], year }}$ & $64.50[61.00,69.00]$ & $63.00[57.00,69.00]$ & $69.00[67.00,69.00]$ & $0.04^{*}$ \\
\hline Sex & & & & $0.03^{*}$ \\
\hline Female, No. (\%) & $11(32.40)$ & $11(42.30)$ & $0(0.00)$ & \\
\hline Male, No. (\%) & $23(67.60)$ & $15(57.70)$ & $8(100.00)$ & \\
\hline $\begin{array}{l}\text { Highest temperature, mean } \pm \mathrm{SD} \text {, } \\
\text { Comorbidities }\end{array}$ & $38.52 \pm 0.73$ & $38.57 \pm 0.73$ & $38.36 \pm 0.76$ & 0.98 \\
\hline Cardiovascular diseases, No. (\%) & $8(23.50)$ & $7(26.90)$ & $1(12.50)$ & 0.65 \\
\hline Hypertension, No. (\%) & $12(35.30)$ & $7(26.90)$ & $5(62.50)$ & 0.10 \\
\hline Diabetes, No. (\%) & $10(29.40)$ & $8(30.80)$ & $2(25.00)$ & 1.00 \\
\hline Chronic kidney disease, No. (\%) & $1(2.90)$ & $1(3.80)$ & $0(0.00)$ & 1.00 \\
\hline COPD, No. $(\%)$ & $1(2.90)$ & $0(0.00)$ & $1(12.50)$ & 0.24 \\
\hline Tuberculosis, No. (\%) & $34(100.0)$ & $26(100.0)$ & $8(100.0)$ & - \\
\hline Malignant tumor, No. (\%) & $1(2.90)$ & $0(0.00)$ & $1(12.50)$ & 0.24 \\
\hline Myoglobin, $\mu \mathrm{g} / \mathrm{L}$ & $67.77[32.25,161.07]$ & $56.93[30.19,98.79]$ & $227.82[195.36 .1,11916.36]$ & $<0.01^{* *}$ \\
\hline Interleukin $6, \mathrm{pg} / \mathrm{ml}$ & $29.05[6.03,78.77]$ & $12.02[5.26,43.70]$ & $78.77[61.13,114.64]$ & $0.01^{*}$ \\
\hline Creatine kinase, U/L & $45.70[27.00,193.38]$ & $53.00[39.00,93.00]$ & $70.00[43.50,345.50]$ & 0.77 \\
\hline D-dimer, $\mu \mathrm{g} / \mathrm{L}$ & $4.75 \pm 6.29$ & $2.70 \pm 4.15$ & $10.89 \pm 7.81$ & $<0.01^{* *}$ \\
\hline Troponin I, ng/mL & $0.018[0.007,0.078]$ & $0.026[0.006,0.087]$ & $0.069[0.048,0.094]$ & $0.02^{*}$ \\
\hline Procalcitonin, $\mathrm{ng} / \mathrm{mL}$ & $0.20[0.13,0.28]$ & $0.22[0.15,0.27]$ & $0.12[0.12,0.15]$ & 0.09 \\
\hline C-reactive protein, mg/L & $64.99 \pm 67.22$ & $49.08 \pm 63.48$ & $121.85 \pm 49.03$ & 0.73 \\
\hline $\mathrm{BNP}, \mathrm{pg} / \mathrm{L}$ & $5.81[2.21,37.11]$ & $3.33[1.79,5.46]$ & $11.23[8.70,63.22]$ & 0.18 \\
\hline LVEF, \% & $62.74 \pm 4.36$ & $62.08 \pm 4.36$ & $64.88 \pm 3.83$ & 0.62 \\
\hline LVDd, mm & $45.50[43.25,48.00]$ & $46.50[44.00,49.00]$ & $40.00[39.50,40.00]$ & 0.14 \\
\hline IVSd, mm & $10.50[10.0,12.00]$ & $10.00[10.00,10.00]$ & $14.00[13.50,15.00]$ & $<0.01^{* *}$ \\
\hline $\mathrm{PaO} 2: \mathrm{FiO} 2$ & $253.33 \pm 122.86$ & $289.52 \pm 106.98$ & $135.74 \pm 98.06$ & 0.58 \\
\hline PASP, mmHg & $33.06 \pm 18.51$ & $24.27 \pm 7.70$ & $61.63 \pm 13.53$ & $<0.01^{*}$ \\
\hline
\end{tabular}

Abbreviations: SD, standard deviation; IQR, interquartile range; $\mathrm{COPD}$, chronic obstructive pulmonary disease; BNP, brain natriuretic peptide; LVEF, left ventricular ejection fraction; LVDd, left ventricular enddiastolic dimension; IVSd, interventricular end-diastolic thickness; PASP, pulmonary artery systolic pressure; SI conversion factors: To convert creatine kinase to $\mu$ kat/L, multiply by $0.0167 .{ }^{*} P<0.05 ;{ }^{* *} P<0.01$. 
Table 2. Binary variables logistic regression analysis

\begin{tabular}{llll}
\hline Predictor & OR & $\mathbf{9 5 \% C I}$ & $P$ value \\
\hline Age, year & 0.88 & $0.75-1.03$ & 0.18 \\
Interleukin 6, pg/mL & 0.99 & $0.97-1.01$ & 0.18 \\
D-dimer, $\mu \mathrm{g} / \mathrm{L}$ & 0.81 & $0.69-0.95$ & $0.01^{*}$ \\
Troponin I, ng/mL & $<0.001$ & $0.00-2.72$ & 0.06 \\
IVSd, mm & 0.16 & $0.045-0.59$ & $<0.01^{*}$ \\
PASP, mmHg & 0.70 & $0.47-1.04$ & 0.08 \\
\hline
\end{tabular}

Abbreviations: OR, odds ratio; CI, confidence interval; IVSd, interventricular end-diastolic thickness; PASP, pulmonary artery systolic pressure; ${ }^{*} P<0.05$.

Table 3. Cox proportional hazard models

\begin{tabular}{lccl}
\hline Predictor & HR & 95\%CI & $P$ value \\
\hline Model 1 & & & $<0.01^{* *}$ \\
PASP, mmHg & 0.94 & $0.90-0.97$ & $<0.01^{* *}$ \\
Model 2 & & & $<0.01^{* *}$ \\
PASP, mmHg & 0.95 & $0.91-0.98$ & $<0.01^{* *}$ \\
D-dimer, $\mu \mathrm{g} / \mathrm{L}$ & 0.88 & $0.78-1.00$ & 0.06 \\
Model 3 & & & $<0.01^{* *}$ \\
PASP, mmHg & 0.94 & $0.89-0.98$ & $<0.01^{* *}$ \\
IVSd, mm & 1.00 & $0.61-1.64$ & 0.99 \\
\hline
\end{tabular}

In Cox multivariate regression model analysis, the outcome was ventilator weaning, the time was days of mechanical respiratory assistance treatment, the start time was the time of admission to the intensive care unit, and the follow-up time was 30 days. PASP has an independent effect on events after adjusting for potential confounders, including time. The adjusted hazard ratio (HR) for $1 \mathrm{mmHg}$ increase in PASP was 0.94 (95\% CI 0.90-0.97) for weaning events $(P<0.001)$. Abbreviations: HR, hazard ratio; CI, confidence interval; PASP, pulmonary artery systolic pressure; IVSd, interventricular end-diastolic thickness; ${ }^{* *} P<$ 0.01 . 

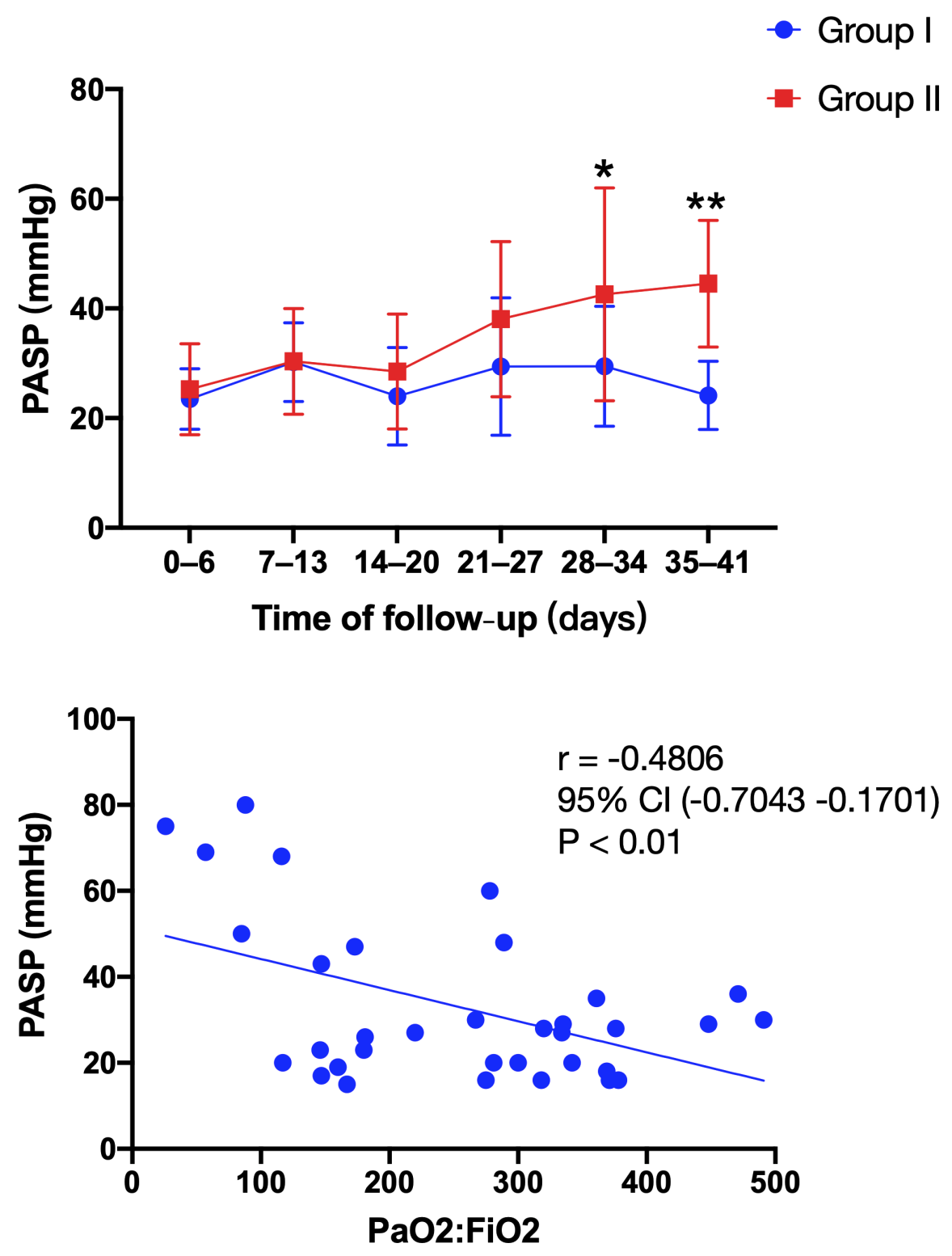

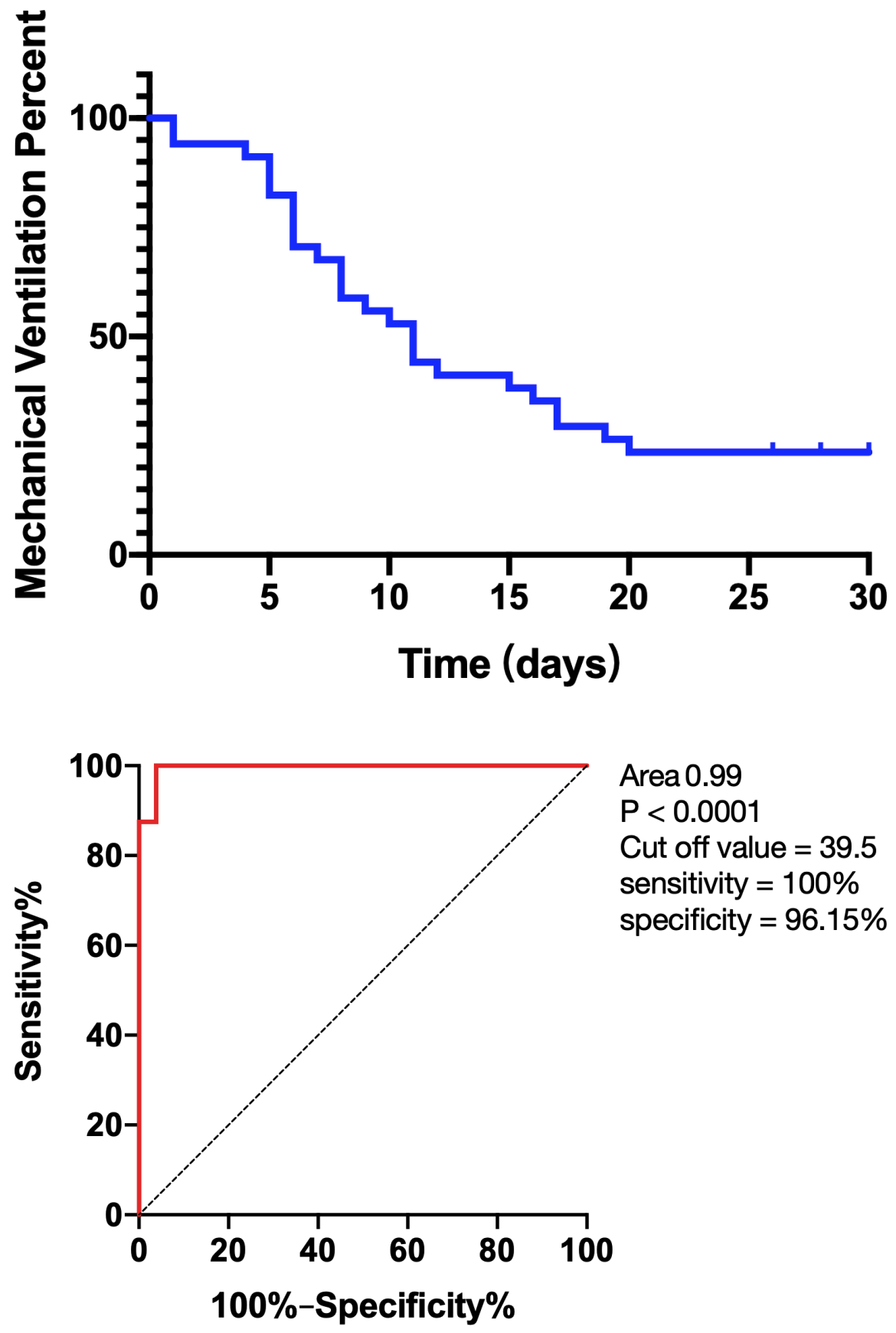